\title{
Time-Dependent MHD Simulations of Jets from Accretion Discs: Stationary Solutions
}

\author{
M.M. Romanova, R.V.E. Lovelace \\ Space Research Institute, Moscow, Russia, and Department of \\ Astronomy, Cornell University, Ithaca, NY 14853-6801 \\ G.V. Ustyugova, A.V. Koldoba, V.M. Chechetkin \\ Keldysh Institute of Applied Mathematics, Moscow, Russia
}

\begin{abstract}
A family of stationary solutions for magnetically driven outflows has been discovered by time-dependent, axisymmetric magnetohydrodynamic simulations of outflows from an accretion disk threaded by an inclined magnetic field. At the disk surface matter was given a small initial push with a speed less than the slow magnetosonic speed. In the simulations, the outflowing matter is observed to be accelerated by magnetic force and by centrifugal force up to velocities in excess of the fast magnetosonic speed and in excess of the escape speed from the central object.
\end{abstract}

\section{Introduction}

Magnetohydrodynamic (MHD) outflows from disks are a promising explanation of the origin of astrophysical jets. Analytic solutions for stationary outflows predict that the field lines at the disk surface diverge away from the symmetry axis. The driving force acting to overcome the gravitational pull of the central object can either be the component of centrifugal force along the magnetic field (Blandford \& Payne 1982) or the magnetic pressure gradient force (Lovelace, et al. 1991).

Axisymmetric numerical simulations of matter outflow from a cold accretion disk performed by Uchida and Shibata (1985) have shown the possibility of non-stationary magnetically driven outflows from an accretion disk. Analogous simulations were performed by Stone and Norman (1994), who concentrated on proccesses inside the disk, and in 3D by Matsumota et al. (1996). In these simulations, the back reaction of the outflow on the disk led to the fast collapse of the disk which prevented investigation of the outflows.

Another approach was taken by Ustyugova et al. (1995), Koldoba et al. (1995), Meier et al. (1996), and Ouyed et al. (1996), who performed 2D numerical simulations of outflows from accretion disks considered as a boundary condition. In this approach it is supposed that the outflow occurs from the relatively hot, rarefied upper layers of the disk without major change of structure of the disk. This allows a study of the outflows during much longer times. However, in the reported studies only temporary outflows were observed. 
Here, we present results of numerical simulations where we succeeded in getting stationary outflows from the disk considered as a boundary condition, starting from non-equilibrium state. Compared with our earlier work (Ustyugova et al. 1995), the magnetic field is much stronger.

\section{Mathematical Model}

We treat time-dependent, axisymmetric MHD flows from upper layers of the accretion disk to the corona. The equations we treat are for ideal, isothermal MHD flows,

$$
\begin{gathered}
\frac{\partial \rho}{\partial t}+\nabla \cdot(\rho \mathbf{v})=0 \\
\frac{\partial \mathbf{v}}{\partial t}+(\mathbf{v} \cdot \nabla) \mathbf{v}+\frac{1}{\rho} \nabla p-\mathbf{g}-\frac{1}{4 \pi \rho}(\nabla \times \mathbf{B}) \times \mathbf{B}=0, \\
\frac{\partial \mathbf{B}}{\partial t}-\nabla \times(\mathbf{v} \times \mathbf{B})=0 .
\end{gathered}
$$

Here, $\rho$ is the mass density, $\mathbf{v}=\left(v_{r}, v_{\phi}, v_{z}\right)$ is the flow velocity, $\mathbf{B}=\left(B_{r}, B_{\phi}, B_{z}\right)$ is magnetic field, $p=\rho c_{s}^{2}$ is the pressure, $c_{s}$ is isothermal sound speed, $\mathrm{g}=-\nabla \Phi_{g}$ is the gravitational acceleration. We smoothed the gravitational potential by taking $\Phi_{g}=-G M / \sqrt{\mathbf{r}^{2}+r_{i}^{2}}$, with $r_{i}$ is the characteristic radius of smoothing which we refer to as the inner radius of the disk. We suppose that plasma outflows from the disk with sub-slow-magnetosonic velocity, and that the disk is perfectly conducting.

The objective of this work was to get stationary super-fast-magnetosonic outflows driven by centrifugal and/or magnetic forces. As we do not know the parameters of the system in the stationary state in advance, we started from nonequilibrium initial conditions: for the gas - equilibrium in the gravitational field, $-(1 / \rho) \nabla p=\mathbf{g}$. For the magnetic field - a "tapered" monopole configuration,

$$
\mathbf{B}_{p}=\mathbf{B}_{m} /\left[1+9 \tan ^{2} \theta\right],
$$

where $\mathbf{B}_{p}$ is poloidal magnetic field, $\mathbf{B}_{m}$ is magnetic field of a monopole, and $\theta$ is an angle between the magnetic field lines and the $z$-axis. Tapering was taken to decrease the collimating effect of the toroidal magnetic field at large $r$ in the disk. The monopole was located under the disk at $z=-2.5 r_{i}$. For the numerical solution of equations (1)-(3), we used a TVD numerical scheme of Godunov's type which was described by Koldoba et al. (1995). We considered the region with $R_{\max }=10 r_{i}, Z_{\max }=15 r_{i}$ and number of grid points $N_{r}=80, N_{z}=120$. The typical run took about 120 hours on a $166 \mathrm{MHz}$ Pentium computer.

\section{RESULTS OF NUMERICAL SIMULATIONS}

Numerical simulations were performed for different parameters $\left(c_{s} / v_{K}\right)_{i}$ and $\left(v_{A} / v_{K}\right)_{i}$, where $v_{A}$ and $v_{K}$ are Alfvénic and Keplerian velocities at radius $r=r_{i}$ at the surface of the disk (not the disk midplane). At the surface of the disk the Alfvén velocity may be comparable or larger than Keplerian velocity. In 


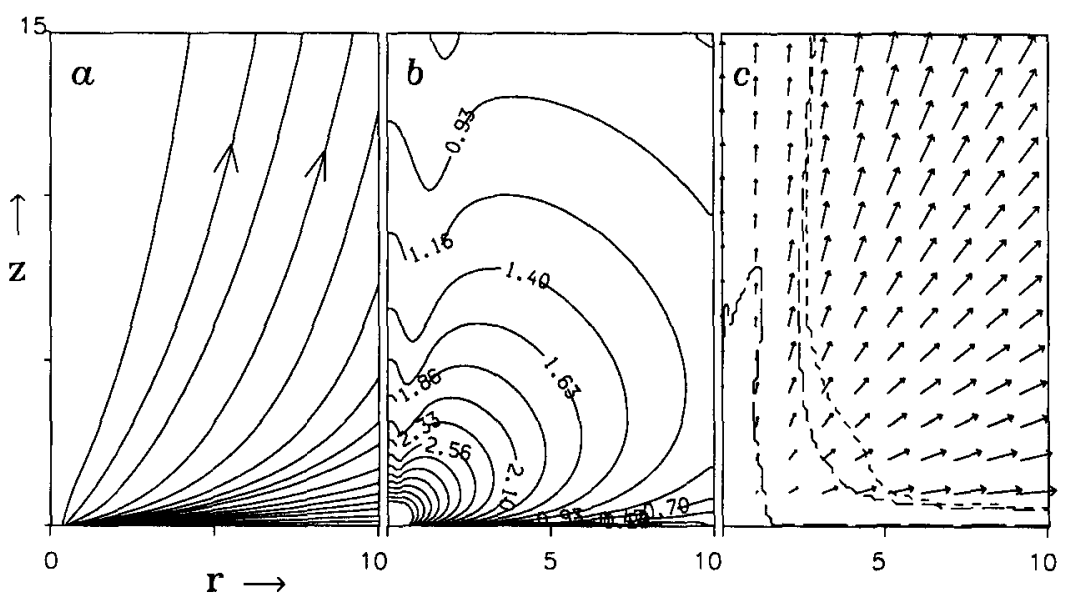

Figure 1. (a) Poloidal field lines at $t=100 t_{i}$. (b) Log of density contours. (c) Velocity vectors of matter flow. Long-dashed line is slow magnetosonic surface, dashed line - Alfvénic surface, and short-dashed line - fast magnetosonic surface.

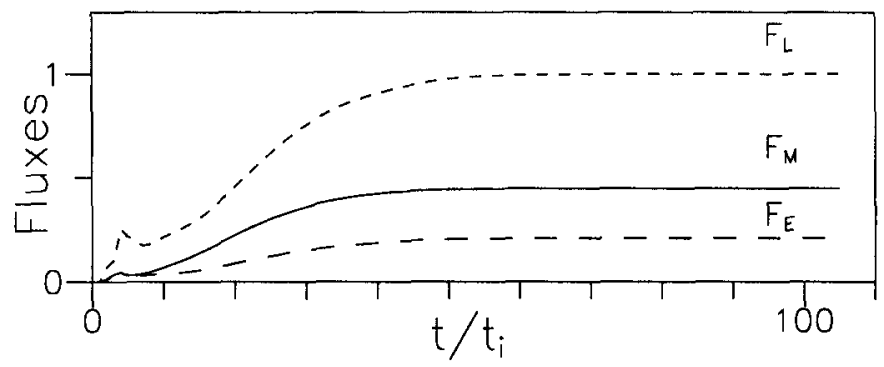

Figure 2. Fluxes through the surface $z=Z_{\max }$ versus time $t . F_{M}$ is the mass flux, $F_{L}$ is the total angular momentum flux; and $F_{E}$ is the total energy flux. The scales are arbitrary. Time is measured in units of $t_{i}$ which is the period of rotation at the inner edge of the disk $\left(r_{i}\right)$.

many cases we got stationary outflows. Figure 1 shows results for one successful run at a late time when the jet is stationary. The flow is accelerated and passes through the slow magnetosonic, Alfvénic, and fast magnetosonic surfaces (Figure 1c). One can see that matter is accelerated basically in the small area near the gravitational center, where the magnetic field is strong. Density contours of the flow are shown at Figure $1 \mathrm{~b}$. Figure 2 shows that matter reaches a stationary state after some period $t \sim 30 t_{i}$ of non-stationary behaviour, where $t_{i}=2 \pi r_{i} / v_{K i}$. Analysis of forces shows, that the main forces driving matter in the area of high inclination of magnetic field lines is a combination of magnetic and centrifugal forces, while in the area around the axis it is thermal pressure gradient force.

We calculated more than 50 different cases with different initial parameters and discovered that in some cases the flow becomes stationary, and in other cases it does not. The area of initial and final parameters of the flow is shown in 


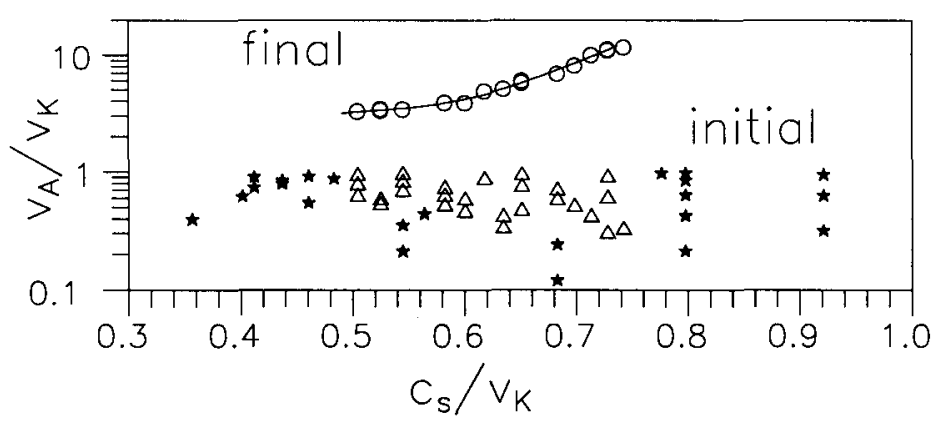

Figure 3. Initial and final parameters of different simulation runs. Triangles are initial parameters of runs which led to stationarity, and stars are for the cases which did not lead to stationarity. Circles are final parameters of stationary runs.

Figure 3. Each point corresponds to separate run. One can see that for a wide range of initial parameters, the final parameters corresponding to stationary outflows are located along a line which is above the initial $v_{A} / v_{K}$ values. Note that all parameters of stationary solutions lie above the line $\left(v_{A} / v_{K}\right)_{i}>3$. It was noticed, that the flow becomes stationary if it passes through the fast magnetosonic surface in the early stages of the simulations.

Acknowledgments. One of the authors (MMR) thanks the IAU for a supporting grant. The authors were supported in part by NSF grant AST-9320068. The Russian authors were also supported in part by the Russian Fundamental Research Foundation and the Program "Astronomy".

\section{References}

Blandford, R.D., \& Payne, D.G. 1982, MNRAS, 199, 883-903

Koldoba, A.V., Ustyugova, G.V., Romanova, M.M., Chechetkin, V.M., \& Lovelace, R.V.E. 1995, Ap\&SS, 232, 241-261

Lovelace, R.V.E., Berk, H.L., \& Contopoulos, J. 1991, ApJ, 379, 696-705

Matsumota, R. et al. 1996, these Proceedings

Meier, D.L., Payne, D.G., \& Lind, K.R. 1995, IAU Symposium N 175, in press

Ouyed, R., Pudritz, R.E., \& Stone, J.M. 1996, Nature, submitted

Stone, J.M., \& Norman, M.L. 1994, ApJ, 433, 746-756

Uchida, Y, \& Shibata, K. 1985, PASJ, 37, 515-535

Ustyugova, G.V., Koldoba A.V., Romanova, M.M., Chechetkin, V.M., \& Lovelace, R.V.E. 1995, ApJ, 439, L39-L42 